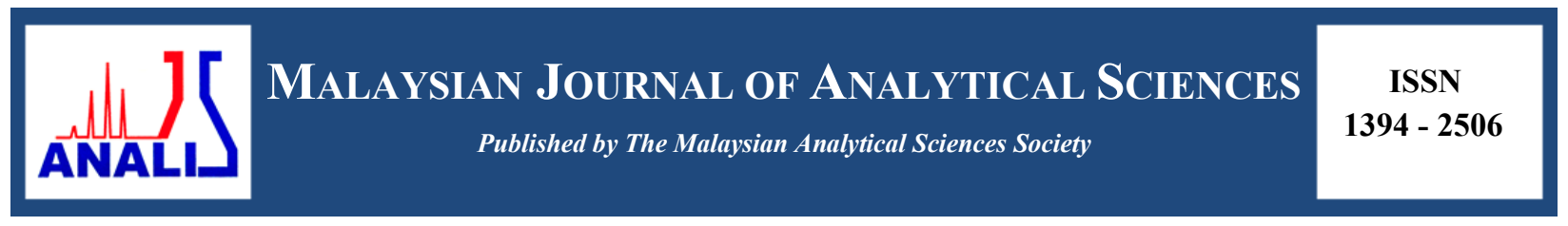

\title{
SYNTHESIS AND CHARACTERIZATION OF OXADIAZOLE DERIVATIVES FROM BENZIMIDAZOLE
}

\author{
(Sintesis dan Perincian Terbitan Oksadiazol Dari Benzimidazol) \\ Norizan Ahmat ${ }^{1,2 *}$, Nik Khairunissa Nik Abdullah Zawawi ${ }^{1,2}$, Muhammad Taha ${ }^{1,2}$, Nor Hadiani Ismail ${ }^{1,2}$, \\ Noraishah Abdullah ${ }^{1}$ \\ ${ }^{I}$ Faculty of Applied Sciences, \\ Universiti Teknologi MARA, 40450 Shah Alam, Selangor, Malaysia \\ ${ }^{2}$ Atta-ur-Rahman Institute for Natural Product Discovery, \\ Universiti Teknologi MARA, Puncak Alam Campus, 42300 Bandar Puncak Alam, Selangor, Malaysia \\ *Corresponding author: noriz118@salam.uitm.edu.my
}

Received: 24 February 2015; Accepted: 27 October 2015

\begin{abstract}
Heterocyclic compounds of benzimidazole and oxadiazole are considered as important class of compounds in medicinal chemistry because of their interesting diversified biological activities. A series of new benzimidazole bearing oxadiazole derivatives have been conveniently synthesized by intermolecular oxidative cyclization of benzimidazole benzoyl hydrazide promoted by iodobenzene diacetate as an oxidant. The structures of the synthesized compounds were characterized by spectroscopic methods namely ${ }^{1} \mathrm{H}$ Nuclear Magnetic Resonance (NMR), Infrared (IR), Mass Spectrometry (MS) and melting point.
\end{abstract}

Keywords: synthesis, benzimidazole, oxadiazole, hydrazide, nuclear magnetic resonance

\section{Abstrak}

Sebatian heterosiklik daripada benzimidazol dan oksadiazol dianggap sebagai satu kelas sebatian penting dalam kimia perubatan kerana aktiviti biologinya yang pelbagai. Satu siri baru terbitan oksadiazol dari benzimidazol telah disintesis dengan mudah oleh siklisasi oksidatif antara molekul daripada benzoil hidrazida benzimidazol dimangkin oleh diasetat iodobenzena sebagai oksidan. Struktur sebatian disintesis telah dicirikan oleh kaedah spektroskopik seperti ${ }^{1} \mathrm{H}$ Resonans Magnet Nukleus (NMR), Inframerah (IR), Spektrometri Jisim (MS) dan takat lebur.

Kata kunci: sintesis, benzimidazol, oxadiazol, hidrazida, resonan megnetik nukleus

\section{Introduction}

Benzimidazole and oxadiazole have received attention in recent years due to widespread applications notably in the field of medicinal chemistry due to their privileged biological activity. Recently, benzimidazole derivatives have been reported as antidiabetic [1], antimicrobial [2-3], antiviral [4-6] antispasmodic [7] and antiasthmatic [8] agents. Similarly, oxadiazole derivatives are also recognized for their pharmacological importance and are reported to possess wide spectrum of activities such as antibacterial [9-11], antifungal [12] anti-inflammatory [13-14] analgesic [15], anticonvulsant [16-17], hypoglycemic [18] and anticancer [19] properties. The synthesis of both compounds has emerged as an essential need for development of new pharmaceutical entities. 


\title{
Norizan et al: SYNTHESIS AND CHARACTERIZATION OF OXADIAZOLE DERIVATIVES FROM
}

BENZIMIDAZOLE

In the present study, a series of novel oxadiazoles were synthesized from benzimidazole and their physical characterization are reported. The structures of the synthesized compounds were characterized using different spectroscopic methods namely ${ }^{1} \mathrm{H}$ Nuclear Magnetic Resonance (NMR), Infrared (IR), Mass Spectrometry (MS) and melting point.

\section{Materials and Methods}

Melting point was taken on Buchi M-560 melting point instrument and was uncorrected. IR spectra were recorded on a Spectrum One FT-IR spectrometer (Perkin Elmer), using KBr discs and values were signified in $\mathrm{cm}^{-1}$. The ${ }^{1} \mathrm{H}$ NMR and ${ }^{13} \mathrm{C}$ NMR spectra were measured on Bruker 500 Ultrashield Plus NMR (500 MHz) in DMSO-d6 as solvent, using tetramethylsilane (TMS) as an internal standard, and chemical shifts are expressed as ppm. ESI MS were determined on Agilent 6330 Ion Trap, using positive/negative mode. HR-ESI-MS were determined on Agilent 6224 TOF-LC/MS using positive mode.

\section{Synthesis of methyl 4-(5,6-dimethyl-1H-benzo[d]imidazol-2-yl)benzoate (a)}

Sodium metasulfite adduct was synthesized according to literature protocol [20]. Equimolar solution of sulphite adduct and 4,5-dimethyl- $O$-phenylenediamine in DMF was refluxed for 6 hours. After the completion of reaction, it was poured into ice water and the solid product (a) was filtered, dried and crystallized.

\section{Synthesis of 4-(5,6-dimethyl-1H-benzo[d]imidazol-2-yl)benzohydrazide (b)}

A solution of compound (a) $(40 \mathrm{mmol})$ in methanol $(50 \mathrm{ml})$ was refluxed for 12 hours in the presence of hydrazine hydrate $(95 \%)$ mixture $(10 \mathrm{ml})$. The reaction mixture was evaporated and the residue was washed with water, filtered, dried, and crystallized.

General procedure for the synthesis of 4-(5,6-dimethyl-1H-benzo[d]imidazol-2-yl) benzohydrazide Schiff bases (1-14)

Equimolar mixture of 4-(5,6-dimethyl-1H-benzo[d]imidazol-2-yl) benzohydrazide $(0.280 \mathrm{~g}, 0.01 \mathrm{~mol})$ selected aldehyde $(0.01 \mathrm{~mol})$, in $n$-butanol $(25 \mathrm{~mL})$ containing acetic acid $(1 \mathrm{~mL})$ was heated under reflux for 3 hours. The reaction mixture was filtered, dried and crystallized.

\section{General procedure for the synthesis of 2,5-disubstituted-1,3,4-oxadiazoles (15-28)}

Compounds 1-14 (100 mg, $\pm 0.25 \mathrm{mmol})$ were dissolved in DMF (5 ml) and iodobenzenediacetate (IBD) ( \pm 0.27 $\mathrm{mmol}$ ) were added [21]. The content was stirred overnight and the progress of reaction was monitored by thin layer chromatography. Cold water was added and the precipitate obtained was filtered, washed with diethyl ether and dried to afford 15-28.

\author{
Results and Discussion \\ Characterization: methyl 4-(5,6-dimethyl-1H-benzo[d]imidazol-2-yl)benzoate (a) \\ Light brown solid; yield (96\%); m.p. $208.9^{\circ} \mathrm{C}$; IR(KBr) $\left(v_{\max }, \mathrm{cm}^{-1}\right): 2954,1720,1273,1107 .{ }^{1} \mathrm{H}$ NMR $(500 \mathrm{MHz}$, \\ DMSO) $\delta: 2.33$ (s, 6H, CH 3 -5,6-benzimidazole), 3.88 (s, 3H, $\mathrm{OCH}_{3}$-benzoate), 7.39 (s, 2H, H-4,7-benzimidazole), \\ 8.09 (d, $J=8.4 \mathrm{~Hz}, 2 \mathrm{H}, \mathrm{Ar}), 8.27$ (d, $J=8.4 \mathrm{~Hz}, 2 \mathrm{H}, \operatorname{Ar})$. ESI MS (m/z): $280.1\left(\mathrm{M}^{+}\right)$.
}

\section{4-(5,6-dimethyl-1H-benzo[d]imidazol-2-yl)benzohydrazide (b)}

Light brown solid; yield ( $93 \%$ ); m.p. $316.5^{\circ} \mathrm{C}$; $\mathrm{IR}(\mathrm{KBr})\left(v_{\max }, \mathrm{cm}^{-1}\right): 3020,1625,1560,1350 .{ }^{1} \mathrm{H} \mathrm{NMR}(500 \mathrm{MHz}$, DMSO) $\delta: 2.33\left(\mathrm{~s}, 6 \mathrm{H}, \mathrm{CH}_{3}-5,6\right.$-benzimidazole), $4.56\left(\mathrm{~s}, 2 \mathrm{H}, \mathrm{NH}_{2}\right), 7.32(\mathrm{~s}, 1 \mathrm{H}, \mathrm{H}-7$-benzimidazole), $7.46(\mathrm{~s}, 1 \mathrm{H}$, H-4-benzimidazole), 7.98 (d, $J=7.5 \mathrm{~Hz}, 2 \mathrm{H}, \mathrm{Ar}), 8.21$ (d, $J=7.5 \mathrm{~Hz}, 2 \mathrm{H}, \mathrm{Ar}), 9.88$ (s, 1H, NH-hydrazide), 12.74 (s, 1H, NH-benzimidazole). ESI MS (m/z): $280.1\left(\mathrm{M}^{+}\right)$.

(E)-4-(5,6-dimethyl-1H-benzo[d]imidazol-2-yl)-N'-(3-fluorobenzylidene ) benzohydrazide (1)

Light brown solid; yield 89\%; m.p. $324.3{ }^{\circ} \mathrm{C}$; IR(KBr) $\left(v_{\max }, \mathrm{cm}^{-1}\right): 3465,1657,1557,1276,1237 .{ }^{1} \mathrm{H}$ NMR $(500$ MHz, DMSO) $\delta: 2.34,2.35$ (s, 6H, $\mathrm{CH}_{3}$-5,6-benzimidazole), 7.31 (m, 2H, Ar), 7.55 (m, 4H, Ar), 8.09 (d, $J=7.7$ $\mathrm{Hz}, 2 \mathrm{H}, \mathrm{Ar}), 8.29$ (d, $J=8.0 \mathrm{~Hz}, 2 \mathrm{H}, \mathrm{Ar}), 8.51(\mathrm{~s}, 1 \mathrm{H}, \mathrm{CH}=\mathrm{N}), 12.05$ (s, 1H, NH-hydrazide), 12.80 (s, 1H, NHbenzimidazole). ESI MS (m/z): $386.2\left(\mathrm{M}^{+}\right)$. 
(E)-4-(5,6-dimethyl-1H-benzo[d]imidazol-2-yl)-N'-(2-hydroxybenzylidene) benzohydrazide (2)

Light brown solid; yield $(90 \%)$; m.p. $352.7{ }^{\circ} \mathrm{C}$; IR(KBr) $\left(v_{\max }, \mathrm{cm}^{-1}\right): 3427,1623,1291 .{ }^{1} \mathrm{H}$ NMR $(500 \mathrm{MHz}$,

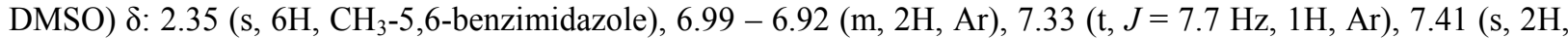
H-4,7-benzimidazole), 7.58 (d, $J=7.4 \mathrm{~Hz}, 1 \mathrm{H}, \mathrm{Ar}), 8.11$ (d, $J=8.2 \mathrm{~Hz}, 2 \mathrm{H}, \mathrm{Ar}$ ), 8.30 (d, $J=8.2 \mathrm{~Hz}, 2 \mathrm{H}, \mathrm{Ar}$ ), 8.69 $(\mathrm{s}, 1 \mathrm{H}, \mathrm{CH}=\mathrm{N}), 11.28(\mathrm{~s}, 1 \mathrm{H}, \mathrm{NH}-h y d r a z i d e), 12.19$ (s, 1H, NH-benzimidazole). ESI MS (m/z): $384.3\left(\mathrm{M}^{-}\right)$.

(E)-4-(5,6-dimethyl-1H-benzo[d]imidazol-2-yl)-N'-(3-hydroxybenzylidene) benzohydrazide (3) Light yellow solid; yield (90\%); m.p. $316.1{ }^{\circ} \mathrm{C}$; IR(KBr) $\left(v_{\max }, \mathrm{cm}^{-1}\right): 3200,1642,1560,1271 .{ }^{1} \mathrm{H}$ NMR $(500 \mathrm{MHz}$, DMSO) $\delta: 2.34$ (s, 6H, CH $3-5,6$-benzimidazole), $7.13(\mathrm{~d}, J=7.2 \mathrm{~Hz}, 1 \mathrm{H}, \mathrm{Ar}), 7.24(\mathrm{~s}, 1 \mathrm{H}, \mathrm{Ar}), 7.28(\mathrm{t}, J=7.7 \mathrm{~Hz}$, $1 \mathrm{H}, \mathrm{Ar}), 7.41$ (s, 2H, H-4,7-benzimidazole), 8.08 (d, $J=7.9 \mathrm{~Hz}, 2 \mathrm{H}, \mathrm{Ar}), 8.28$ (d, $J=8.0 \mathrm{~Hz}, 2 \mathrm{H}, \mathrm{Ar}), 8.41(\mathrm{~s}, 1 \mathrm{H}$, $\mathrm{CH}=\mathrm{N}), 9.66(\mathrm{~s}, 1 \mathrm{H}, \mathrm{OH}), 11.88(\mathrm{~s}, 1 \mathrm{H}, \mathrm{NH}$-hydrazide), $12.80(\mathrm{~d}, J=1.0 \mathrm{~Hz}, 1 \mathrm{H}, \mathrm{NH}-$ benzimidazole). ESI MS $(\mathrm{m} / \mathrm{z}): 384.3\left(\mathrm{M}^{-}\right)$.

(E)-N'-(3,4-dimethoxybenzylidene)-4-(5,6-dimethyl-1H-benzo[d]imidazol-2-yl) benzohydrazide (4) Light yellow solid; yield (92\%); m.p. $316.4{ }^{\circ} \mathrm{C}$; $\mathrm{IR}(\mathrm{KBr})\left(v_{\max }, \mathrm{cm}^{-1}\right): 3434,1658,1564,1272 .{ }^{1} \mathrm{H} \mathrm{NMR}(500 \mathrm{MHz}$,

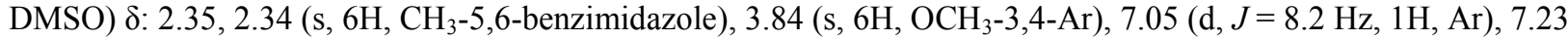
$(\mathrm{d}, J=7.8 \mathrm{~Hz}, 1 \mathrm{H}, \mathrm{Ar}), 7.33(\mathrm{~s}, 1 \mathrm{H}, \mathrm{Ar}), 7.38(\mathrm{~s}, 1 \mathrm{H}, \mathrm{Ar}), 7.47(\mathrm{~s}, 1 \mathrm{H}, \mathrm{Ar}), 8.07$ (d, $J=8.1 \mathrm{~Hz}, 2 \mathrm{H}, \mathrm{Ar}), 8.28(\mathrm{~d}, J=$ $8.1 \mathrm{~Hz}, 2 \mathrm{H}, \mathrm{Ar}), 8.42(\mathrm{~s}, 1 \mathrm{H}, \mathrm{CH}=\mathrm{N}), 11.83$ (s, 1H, NH-hydrazide), 12.81 (s, 1H, NH-benzimidazole). ESI MS $(\mathrm{m} / \mathrm{z}): 428.3\left(\mathrm{M}^{+}\right)$.

(E)-4-(5,6-dimethyl-1H-benzo[d]imidazol-2-yl)-N'-(4-nitrobenzylidene) benzohydrazide (5)

Light yellow solid; yield (92\%); m.p. $323.6{ }^{\circ} \mathrm{C}$; IR $(\mathrm{KBr})\left(v_{\max }, \mathrm{cm}^{-1}\right): 3269,1637,1560,1519,1343,1282 .{ }^{1} \mathrm{H}$ NMR (500 MHz, DMSO) $\delta: 2.33,2.35$ (s, 6H, $\mathrm{CH}_{3}-5,6$-benzimidazole), 7.32 (s, 1H, H-7), 7.46 (s, 1H, H-4), 8.00 (d, $J=$ $8.1 \mathrm{~Hz}, 2 \mathrm{H}, \mathrm{Ar}), 8.09$ (d, $J=7.7 \mathrm{~Hz}, 2 \mathrm{H}, \mathrm{Ar}), 8.32-8.27$ (m, 4H, Ar), 8.57 (s, 1H, CH=N), 12.23 (s, 1H, NHhydrazide), 12.83 (s, 1H, NH-benzimidazole). ESI MS (m/z): $413.3\left(\mathrm{M}^{+}\right)$.

(E)-4-(5,6-dimethyl-1H-benzo[d]imidazol-2-yl)-N'-(4-fluorobenzylidene) benzohydrazide (6) Light brown solid; yield (94\%); m.p. $330.7^{\circ} \mathrm{C}$; IR( $\left.\mathrm{KBr}\right)\left(v_{\max }, \mathrm{cm}^{-1}\right)$ : 3256, 1630, 1287, 1236. ${ }^{1} \mathrm{H}$ NMR $(500 \mathrm{MHz}$, DMSO) $\delta: 2.34$ (s, 6H, CH $-5,6$-benzimidazole), 7.32 (t, $J=8.6 \mathrm{~Hz}, 2 \mathrm{H}, \mathrm{Ar}), 7.40$ (s, 2H, Ar), $7.87-7.78$ (m, 2H, Ar), $8.08(\mathrm{~d}, J=8.0 \mathrm{~Hz}, 2 \mathrm{H}, \operatorname{Ar}), 8.28(\mathrm{~d}, J=8.1 \mathrm{~Hz}, 2 \mathrm{H}, \operatorname{Ar}), 8.49(\mathrm{~s}, 1 \mathrm{H}, \mathrm{CH}=\mathrm{N}), 11.96(\mathrm{~s}, 1 \mathrm{H}, \mathrm{NH}-$ benzimidazole). ESI MS (m/z): $386.9\left(\mathrm{M}^{-}\right)$.

(E)-4-(5,6-dimethyl-1H-benzo[d]imidazol-2-yl)-N'-(4-methoxybenzylidene) benzohydrazide (7)

Light brown solid; yield (91\%); m.p. $276.0^{\circ} \mathrm{C}$; $\mathrm{IR}(\mathrm{KBr})\left(v_{\max }, \mathrm{cm}^{-1}\right): 3234,1611,1512,1258 .{ }^{1} \mathrm{H}$ NMR $(500 \mathrm{MHz}$, DMSO) $\delta: 2.34$ (s, 6H, $\mathrm{CH}_{3}-5,6$-benzimidazole), $3.82\left(\mathrm{~s}, 3 \mathrm{H}, \mathrm{OCH}_{3}\right), 7.04$ (d, J=8.6 Hz, 2H, Ar), $7.51-7.29$ (m, $2 \mathrm{H}, \operatorname{Ar}), 7.71(\mathrm{~d}, J=8.5 \mathrm{~Hz}, 2 \mathrm{H}, \mathrm{Ar}), 8.08(\mathrm{~d}, J=8.2 \mathrm{~Hz}, 2 \mathrm{H}, \mathrm{Ar}), 8.28(\mathrm{~d}, J=8.2 \mathrm{~Hz}, 2 \mathrm{H}, \mathrm{Ar}), 8.44(\mathrm{~s}, 1 \mathrm{H}$, $\mathrm{CH}=\mathrm{N}), 11.83$ (s, 1H, NH-benzimidazole). ESI MS (m/z): $398.9\left(\mathrm{M}^{-}\right)$.

(Z)-N'-(4-chlorobenzylidene)-4-(5,6-dimethyl-1H-benzo[d]imidazol-2-yl)benzohydrazide (8)

Light brown solid; yield (90\%); m.p. $338.7^{\circ} \mathrm{C}$; $\mathrm{IR}(\mathrm{KBr})\left(v_{\max }, \mathrm{cm}^{-1}\right): 3253,1634,1565,1286,669 .{ }^{1} \mathrm{H}$ NMR $(500$ MHz, DMSO) $\delta:$ 2.34, 2.36 (s, 6H, $\mathrm{CH}_{3}$-5,6-benzimidazole), 7.33 (s, 1H, Ar), 7.47 (s, 1H, Ar), 7.55 (d, $J=8.0 \mathrm{~Hz}$, $2 \mathrm{H}, \mathrm{Ar}), 7.79(\mathrm{~d}, J=8.1 \mathrm{~Hz}, 2 \mathrm{H}, \mathrm{Ar}), 8.08$ (d, $J=7.9 \mathrm{~Hz}, 2 \mathrm{H}, \mathrm{Ar}), 8.28(\mathrm{~d}, J=8.0 \mathrm{~Hz}, 2 \mathrm{H}, \mathrm{Ar}), 8.49(\mathrm{~s}, 1 \mathrm{H}$, $\mathrm{CH}=\mathrm{N}), 12.01$ (s, 1H, NH-hydrazide), 12.80 (s, 1H, NH-benzimidazole). ESI MS (m/z): $402.3\left(\mathrm{M}^{+}\right)$.

(E)-4-(5,6-dimethyl-1H-benzo[d]imidazol-2-yl)-N'-(pyridin-4-ylmethylene) benzohydrazide (9)

Light brown solid; yield (90\%); m.p. $306.7^{\circ} \mathrm{C}$; $\operatorname{IR}(\mathrm{KBr})\left(v_{\max }, \mathrm{cm}^{-1}\right): 3277,1634,1560,1288 .{ }^{1} \mathrm{H}$ NMR $(500 \mathrm{MHz}$, DMSO) $\delta: 2.33$ (s, 6H, CH $-5,6$-benzimidazole), 7.40 (s, 2H, Ar), 7.69 (s, 2H, Ar), 8.10 (d, J= 7.2 Hz, 2H, Ar), $8.30(\mathrm{~d}, J=7.9 \mathrm{~Hz}, 2 \mathrm{H}, \mathrm{Ar}), 8.48(\mathrm{~s}, 1 \mathrm{H}, \mathrm{CH}=\mathrm{N}), 8.67(\mathrm{~s}, 2 \mathrm{H}, \mathrm{Ar}), 12.22(\mathrm{~s}, 1 \mathrm{H}, \mathrm{NH}-$ benzimidazole). ESI MS $(\mathrm{m} / \mathrm{z}): 369.3\left(\mathrm{M}^{+}\right)$.

(E)-N'-(3-chlorobenzylidene)-4-(5,6-dimethyl-1H-benzo[d]imidazol-2-yl) benzohydrazide (10)

Light brown solid; yield $(91 \%)$; m.p. $358.5^{\circ} \mathrm{C}$; $\mathrm{IR}(\mathrm{KBr})\left(v_{\max }, \mathrm{cm}^{-1}\right): 3422(\mathrm{NH}), 1651,1564,1282,704 .{ }^{1} \mathrm{H}$ NMR (500 MHz, DMSO) $\delta: 2.33$ (s, 6H, CH $-5,6$-benzimidazole), 7.40 (s, 2H, Ar), 7.49 (s, 2H, Ar), 7.71 (s, 1H, Ar), 7.81 
Norizan et al: SYNTHESIS AND CHARACTERIZATION OF OXADIAZOLE DERIVATIVES FROM

BENZIMIDAZOLE

(s, 1H, Ar), 8.09 (d, $J=7.6 \mathrm{~Hz}, 2 \mathrm{H}, \mathrm{Ar}), 8.29$ (d, $J=7.8 \mathrm{~Hz}, 2 \mathrm{H}, \mathrm{Ar}), 8.47$ (s, 1H, CH=N), 12.09 (s, 1H, NHbenzimidazole). ESI MS (m/z): $402.3\left(\mathrm{M}^{+}\right)$.

(E)-4-(5,6-dimethyl-1H-benzo[d]imidazol-2-yl)-N'-(2-methylbenzylidene) benzohydrazide (11)

Light brown solid; yield (93\%); m.p. $323.8^{\circ} \mathrm{C}$; IR( $\left.\mathrm{KBr}\right)\left(v_{\max }, \mathrm{cm}^{-1}\right): 3057(\mathrm{NH}), 1637,1275 .{ }^{1} \mathrm{H}$ NMR $(500 \mathrm{MHz}$,

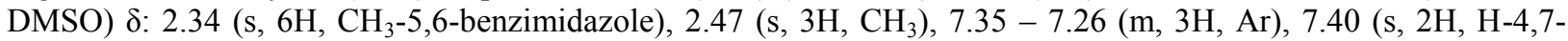
benzimidazole), $7.89(\mathrm{~d}, J=7.3 \mathrm{~Hz}, 1 \mathrm{H}, \mathrm{Ar}), 8.10(\mathrm{~d}, J=8.0 \mathrm{~Hz}, 2 \mathrm{H}, \mathrm{Ar}), 8.29(\mathrm{~d}, J=8.0 \mathrm{~Hz}, 2 \mathrm{H}, \mathrm{Ar}), 8.80(\mathrm{~s}, 1 \mathrm{H}$, $\mathrm{CH}=\mathrm{N}), 11.92$ (s, 1H, NH-benzimidazole). ESI MS (m/z): $382.3\left(\mathrm{M}^{+}\right)$.

(E)-4-(5,6-dimethyl-1H-benzo[d]imidazol-2-yl)-N'-(4 hydroxybenzylidene) benzohydrazide (12)

Light yellow solid; yield (91\%); m.p. $313.4^{\circ} \mathrm{C}$; $\mathrm{IR}(\mathrm{KBr})\left(v_{\max }, \mathrm{cm}^{-1}\right): 3198(\mathrm{NH}), 1610,1272 .{ }^{1} \mathrm{H}$ NMR $(500 \mathrm{MHz}$, DMSO) $\delta: 2.35\left(\mathrm{~s}, 6 \mathrm{H}, \mathrm{CH}_{3}-5,6\right), 6.86(\mathrm{~d}, J=8.2 \mathrm{~Hz}, 2 \mathrm{H}, \mathrm{Ar}), 7.40$ (s, 2H, H-4,7-benzimidazole), 7.59 (d, $J=8.2$ $\mathrm{Hz}, 2 \mathrm{H}, \mathrm{Ar}), 8.07$ (d, $J=8.1 \mathrm{~Hz}, 2 \mathrm{H}, \mathrm{Ar}), 8.27(\mathrm{~d}, J=8.1 \mathrm{~Hz}, 2 \mathrm{H}, \mathrm{Ar}), 8.39$ (s, 1H, CH=N), 9.95 (s, $1 \mathrm{H}, \mathrm{NH}-$ hydrazide), 11.74 (s, 1H, NH-benzimidazole). ESI MS (m/z): $384.3\left(\mathrm{M}^{+}\right)$.

(E)-4-(5,6-dimethyl-1H-benzo[d]imidazol-2-yl)-N'-(pyridin-3-ylmethylene) benzohydrazide (13)

Light yellow solid; yield (92\%); m.p. $348.3{ }^{\circ} \mathrm{C}$; IR( $\left.\mathrm{KBr}\right)\left(v_{\max }, \mathrm{cm}^{-1}\right): 3194,1647,1552,1281 .{ }^{1} \mathrm{H}$ NMR $(500 \mathrm{MHz}$, DMSO) $\delta: 2.33$ (s, 6H, CH 3 -5,6-benzimidazole), $7.40(\mathrm{~s}, 2 \mathrm{H}, \mathrm{Ar}), 7.50(\mathrm{~s}, 1 \mathrm{H}, \mathrm{Ar}), 8.10(\mathrm{~d}, J=7.5 \mathrm{~Hz}, 2 \mathrm{H}, \mathrm{Ar})$, 8.17 (d, $J=6.9 \mathrm{~Hz}, 1 \mathrm{H}, \mathrm{Ar}), 8.30$ (d, $J=7.7 \mathrm{~Hz}, 2 \mathrm{H}, \mathrm{Ar}), 8.55$ (s, 1H, Ar), $8.63(\mathrm{~s}, 1 \mathrm{H}, \mathrm{Ar}), 8.89$ (s, 1H, CH=N), 12.12 (s, 1H, NH-hydrazide), 12.81 (s, 1H, NH-benzimidazole). ESI MS (m/z): $369.3\left(\mathrm{M}^{+}\right)$.

(E)-4-(5,6-dimethyl-1H-benzo[d]imidazol-2-yl)-N'-(3 methylbenzylidene) benzohydrazide (14)

White solid; yield $(93 \%)$; m.p. $321.2{ }^{\circ} \mathrm{C}$; IR( $\left.\mathrm{KBr}\right)\left(v_{\max }, \mathrm{cm}^{-1}\right): 3422,1656,1564,1283 .{ }^{1} \mathrm{H}$ NMR (500 MHz,

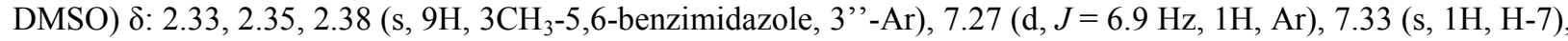
7.37 (t, $J=7.3 \mathrm{~Hz}, 1 \mathrm{H}, \mathrm{Ar}), 7.47$ (s, 1H, H-4), 7.54 (d, $J=7.1 \mathrm{~Hz}, 1 \mathrm{H}, \mathrm{Ar}), 7.59$ (s, 1H, H-2')), 8.08 (d, $J=7.6 \mathrm{~Hz}$, $2 \mathrm{H}, \mathrm{Ar}), 8.28(\mathrm{~d}, J=7.8 \mathrm{~Hz}, 2 \mathrm{H}, \mathrm{Ar}), 8.46(\mathrm{~s}, 1 \mathrm{H}, \mathrm{CH}=\mathrm{N}), 11.93(\mathrm{~s}, 1 \mathrm{H}, \mathrm{NH}$-hydrazide), $12.82(\mathrm{~s}, 1 \mathrm{H}, \mathrm{NH}-$ benzimidazole). ESI MS (m/z): $382.3\left(\mathrm{M}^{-}\right)$.

2-(4-(5,6-dimethyl-1H-benzo[d]imidazol-2-yl)phenyl)-5-(3-fluorophenyl)-1,3,4-oxadiazole (15)

Pale yellow powder, yield: $0.075 \mathrm{~g}(75 \%)$, m.p.: $346.5^{\circ} \mathrm{C}$. IR $(\mathrm{KBr})\left(v_{\max }, \mathrm{cm}^{-1}\right): 3308,1611,1317,1262,1070$,

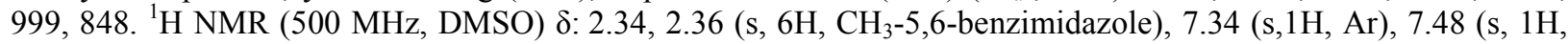
Ar), $7.54(\mathrm{td}, 1 \mathrm{H}, J=8.6,1.9, \mathrm{Ar}), 7.55(\mathrm{t}, 1 \mathrm{H}, J=7.6 \mathrm{~Hz}, \mathrm{Ar}), 7.72(\mathrm{dd}, 1 \mathrm{H}, J=13.8,8.0 \mathrm{~Hz}, \mathrm{Ar}), 8.03(\mathrm{dd}, 2 \mathrm{H}, J$ $=15.7,8.6 \mathrm{~Hz}, \mathrm{Ar}), 8.33$ (d, 2H, $J=8.5 \mathrm{~Hz}, \mathrm{Ar}), 8.38$ (d, 2H, $J=8.4 \mathrm{~Hz}, \mathrm{Ar}), 12.9$ (s, 1H, NH). HR-ESI-MS: m/z $384.2288\left(\mathrm{M}^{+}\right)$.

2-(5-(4-(5,6-dimethyl-1H-benzo[d]imidazol-2-yl)phenyl)-1,3,4-oxadiazol-2-yl)phenol (16)

Pale yellow powder, yield: $0.081 \mathrm{~g}(81 \%)$, m.p.: $375.7^{\circ} \mathrm{C}$; IR $(\mathrm{KBr})\left(v_{\max }, \mathrm{cm}^{-1}\right): 3235,1612,1317,1256,1155$, 1062. ${ }^{1} \mathrm{H}$ NMR (500 MHz, DMSO) $\delta: 2.33,2.36$ (s, 6H, $\mathrm{CH}_{3}$-5,6-benzimidazole), $7.18-7.05$ (m, 2H, Ar), 7.34 (s, $1 \mathrm{H}, \mathrm{Ar}), 7.56-7.42(\mathrm{~m}, 2 \mathrm{H}, \mathrm{Ar}), 7.97(\mathrm{dt}, 1 \mathrm{H}, J=10.1,5.1 \mathrm{~Hz}, \mathrm{Ar}), 8.26(\mathrm{~d}, 2 \mathrm{H}, J=8.5 \mathrm{~Hz}, \mathrm{Ar}), 8.38(\mathrm{~d}, 2 \mathrm{H}, J=$ $8.5 \mathrm{~Hz}, \mathrm{Ar}), 10.36(\mathrm{~s}, 1 \mathrm{H}, \mathrm{OH}), 12.89(\mathrm{~s}, 1 \mathrm{H}, \mathrm{NH})$. HR-ESI-MS: m/z $382.2325\left(\mathrm{M}^{+}\right)$.

3-(5-(4-(5,6-dimethyl-1H-benzo[d]imidazol-2-yl)phenyl)-1,3,4-oxadiazol-2-yl)phenol (17)

Pale yellow powder, yield: $0.082 \mathrm{~g} \mathrm{(82 \% );} \mathrm{m.p.:} 369.9^{\circ} \mathrm{C}$; IR $(\mathrm{KBr})\left(v_{\max }, \mathrm{cm}^{-1}\right): 3236,1610,1315,1262,1069$, 1008. ${ }^{1} \mathrm{H}$ NMR (500 MHz, DMSO) $\delta: 2.33$ (s, 6H, $\mathrm{CH}_{3}-5,6$-benzimidazole), 7.06 (d, $\left.1 \mathrm{H}, J=6.6 \mathrm{~Hz}, \mathrm{Ar}\right), 7.40$ (s, $2 \mathrm{H}, \mathrm{Ar}), 7.45$ (t, $1 \mathrm{H}, J=7.8 \mathrm{~Hz}, \mathrm{Ar}), 7.54$ (s, $1 \mathrm{H}, \mathrm{Ar}), 7.59$ (d, 1H, $J=7.5 \mathrm{~Hz}, \mathrm{Ar}), 8.25$ (d, 2H, $J=8.3 \mathrm{~Hz}, \mathrm{Ar}$ ), $8.35(\mathrm{~d}, 2 \mathrm{H}, J=8.3 \mathrm{~Hz}, \mathrm{Ar}), 10.04$ (s, 1H, OH). HR-ESI-MS: $\mathrm{m} / \mathrm{z} 382.1843\left(\mathrm{M}^{+}\right)$.

\section{2-(3,4-dimethoxyphenyl)-5-(4-(5,6-dimethyl-1H-benzo[d]imidazol-2-yl)phenyl)-1,3,4-oxadiazole (18)}

Pale yellow powder, yield: $0.076 \mathrm{~g}(76 \%)$; m.p.: $293.4^{\circ} \mathrm{C}$; IR $(\mathrm{KBr})\left(v_{\max }, \mathrm{cm}^{-1}\right): 3255,1611,1315,1275,1106$, 1025. 'H NMR (500 MHz, DMSO) $\delta: 2.33,2.35$ (s, 6H, CH $\mathrm{CH}_{3}-5,6$-benzimidazole), 3.88, 3.92 (s, $6 \mathrm{H}, \mathrm{OCH}_{3}-3$ "',4"'$\operatorname{Ar}), 7.20$ (d, 1H, $J=8.5 \mathrm{~Hz}, \mathrm{Ar}), 7.39$ (s, $2 \mathrm{H}, \mathrm{Ar}), 7.65$ (d, $1 \mathrm{H}, J=1.9 \mathrm{~Hz}, \mathrm{Ar}), 7.76$ (dd, $1 \mathrm{H}, J=8.4,2.0 \mathrm{~Hz}, \mathrm{Ar}$ ), 8.28 (d, 2H, $J=8.5 \mathrm{~Hz}, \mathrm{Ar}), 8.36$ (d, 2H, $J=8.5 \mathrm{~Hz}, \mathrm{Ar}), 12.87$ (s, 1H, NH). HR-ESI-MS: m/z $426.2690\left(\mathrm{M}^{+}\right)$. 
2-(4-(5,6-dimethyl-1H-benzo[d]imidazol-2-yl)phenyl)-5-(4-nitrophenyl)-1,3,4-oxadiazole (19)

Pale yellow powder, yield: $0.076 \mathrm{~g}(76 \%)$; m.p.: $361.2^{\circ} \mathrm{C}$; IR $(\mathrm{KBr})\left(v_{\max }, \mathrm{cm}^{-1}\right): 3302,1612,1523,1340,1264$,

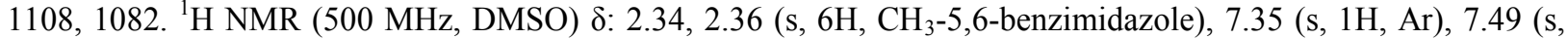
$1 \mathrm{H}, \mathrm{Ar}), 8.33$ (d, 2H, $J=8.4 \mathrm{~Hz}, \mathrm{Ar}), 8.40$ (d, 2H, $J=8.4 \mathrm{~Hz}, \mathrm{Ar}), 8.45,8.48$ (d, 4H, $J=8.9 \mathrm{~Hz}, \mathrm{Ar}), 12.90$ (s, $1 \mathrm{H}$, NH). HR-ESI-MS: m/z $411.2255\left(\mathrm{M}^{+}\right)$.

2-(4-(5,6-dimethyl-1H-benzo[d]imidazol-2-yl)phenyl)-5-(4-fluorophenyl)-1,3,4-oxadiazole (20)

Pale yellow powder, yield: $0.083 \mathrm{~g}(83 \%)$; m.p.: $372.7^{\circ} \mathrm{C}$; IR $(\mathrm{KBr})\left(v_{\max }, \mathrm{cm}^{-1}\right): 3292,1610,1318,1264,1164$, 1002, 845. ${ }^{1} \mathrm{H}$ NMR $(500 \mathrm{MHz}, \mathrm{DMSO}) \delta: 2.34,2.36$ (s, 6H, $\mathrm{CH}_{3}-5$,6-benzimidazole), 7.34 (s, $\left.1 \mathrm{H}, \mathrm{Ar}\right), 7.49$ (d, $2 \mathrm{H}$, $J=9.0 \mathrm{~Hz}, \mathrm{Ar}), 7.54$ (d, $2 \mathrm{H}, J=9.0 \mathrm{~Hz}, \mathrm{Ar}), 8.26(\mathrm{dd}, 2 \mathrm{H}, J=8.8,5.4 \mathrm{~Hz}, \mathrm{Ar}), 8.30$ (d, $2 \mathrm{H}, J=8.5 \mathrm{~Hz}, \mathrm{Ar}), 8.38$ (d, $2 \mathrm{H}, J=8.5 \mathrm{~Hz}, \mathrm{Ar}), 12.90$ (s, 1H, NH). HR-ESI-MS: m/z $384.2288\left(\mathrm{M}^{+}\right)$.

2-(4-(5,6-dimethyl-1H-benzo[d]imidazol-2-yl)phenyl)-5-(4-methoxyphenyl)-1,3,4-oxadiazole (21)

Pale yellow powder, yield: $0.077 \mathrm{~g}(77 \%)$; m.p.: $342{ }^{\circ} \mathrm{C}$; IR $(\mathrm{KBr})\left(v_{\max }, \mathrm{cm}^{-1}\right): 3244,1614,1311,1263,1174,1033$.

${ }^{1} \mathrm{H}$ NMR (500 MHz, DMSO) $\delta: 2.34,2.36$ (s, 6H, $\mathrm{CH}_{3}-5,6$-benzimidazole), 3.89 (s, $3 \mathrm{H}, \mathrm{OCH}_{3}-4$ '”-Ar), 7.20 (d, $2 \mathrm{H}$, $J=8.8 \mathrm{~Hz}, \mathrm{Ar}), 7.34$ (s, $1 \mathrm{H}, \mathrm{Ar}), 7.48$ (s, $1 \mathrm{H}, \mathrm{Ar}), 8.12$ (d, $2 \mathrm{H}, J=8.8 \mathrm{~Hz}, \mathrm{Ar}), 8.28$ (d, 2H, $J=8.4 \mathrm{~Hz}, \mathrm{Ar}), 8.37$ (d, $2 \mathrm{H}, J=8.5 \mathrm{~Hz}, \mathrm{Ar}), 12.89$ (s, 1H, NH). HR-ESI- MS: m/z $396.2500\left(\mathrm{M}^{+}\right)$.

2-(4-chlorophenyl)-5-(4-(5,6-dimethyl-1H-benzo[d]imidazol-2-yl)phenyl)-1,3,4-oxadiazole (22)

Pale yellow powder, yield: $0.075 \mathrm{~g}(75 \%)$; m.p.: $369.7^{\circ} \mathrm{C}$; IR $(\mathrm{KBr})\left(v_{\max }, \mathrm{cm}^{-1}\right): 3280,1611,1317,1264,1093$, 1016, 844. ${ }^{1} \mathrm{H}$ NMR $(500 \mathrm{MHz}, \mathrm{DMSO}) \delta: 2.34,2.36$ (s, 6H, $\mathrm{CH}_{3}-5$,6-benzimidazole), 7.35 (s, $\left.1 \mathrm{H}, \mathrm{Ar}\right), 7.48(\mathrm{~s}, 1 \mathrm{H}$, Ar), $7.74(\mathrm{~d}, 2 \mathrm{H}, J=8.5 \mathrm{~Hz}, \mathrm{Ar}), 8.20(\mathrm{~d}, 2 \mathrm{H}, J=8.5 \mathrm{~Hz}, \mathrm{Ar}), 8.30$ (d, 2H, $J=8.4 \mathrm{~Hz}, \mathrm{Ar}), 8.37$ (d, $2 \mathrm{H}, J=8.4 \mathrm{~Hz}$,

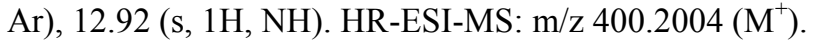

2-(4-(5,6-dimethyl-1H-benzo[d]imidazol-2-yl)phenyl)-5-(pyridin-4-yl)-1,3,4-oxadiazole (23)

Pale yellow powder, yield: $0.068 \mathrm{~g}(68 \%)$; m.p.: $373.0^{\circ} \mathrm{C}$; IR $(\mathrm{KBr})\left(v_{\max }, \mathrm{cm}^{-1}\right): 3051,1612,1571,1321,1272$, 1061, 1002. ${ }^{1} \mathrm{H}$ NMR (500 MHz, DMSO $\delta: 2.35$ (s, 6H, $\mathrm{CH}_{3}-5,6$-benzimidazole), 7.41 (s, 2H, Ar), 8.10 (d, $2 \mathrm{H}, J=$ $5.5 \mathrm{~Hz}, \mathrm{Ar}), 8.33$ (d, 2H, $J=8.4 \mathrm{~Hz}, \mathrm{Ar}), 8.39$ (d, 2H, $J=8.4 \mathrm{~Hz}, \mathrm{Ar}), 8.89$ (d, 2H, $J=4 \mathrm{~Hz}, \mathrm{Ar})$. HR-ESI-MS: m/z $367.1948\left(\mathrm{M}^{+}\right)$.

2-(3-chlorophenyl)-5-(4-(5,6-dimethyl-1H-benzo[d]imidazol-2-yl)phenyl)-1,3,4-oxadiazole (24)

Pale yellow powder, yield: $0.071 \mathrm{~g}(71 \%)$; m.p.: $356.2^{\circ} \mathrm{C}$; IR $(\mathrm{KBr})\left(v_{\max }, \mathrm{cm}^{-1}\right): 3053,1614,1316,1266,1032$, 1003, 847. ${ }^{1} \mathrm{H}$ NMR (500 MHz, DMSO) $\delta: 2.33,2.35$ (s, 6H, $\mathrm{CH}_{3}-5,6$-benzimidazole), 7.34 (s, $1 \mathrm{H}, \mathrm{Ar}$ ), 7.48 (s, $1 \mathrm{H}$, Ar), 7.69 (t, $1 \mathrm{H}, J=8,7.5 \mathrm{~Hz}, \mathrm{Ar}), 7.75(\mathrm{~d}, 1 \mathrm{H}, J=8 \mathrm{~Hz}, \mathrm{Ar}), 8.14$ (d, $1 \mathrm{H}, J=7.5 \mathrm{~Hz}, \mathrm{Ar}), 8.22(\mathrm{~s}, 1 \mathrm{H}, \mathrm{Ar}), 8.32$ (d, $2 \mathrm{H}, J=8 \mathrm{~Hz}, \mathrm{Ar}), 8.37$ (d, 2H, $J=8 \mathrm{~Hz}, \mathrm{Ar}), 12.89$ (s, 1H, NH). HR-ESI-MS: m/z $400.2009\left(\mathrm{M}^{+}\right)$.

2-(4-(5,6-dimethyl-1H-benzo[d]imidazol-2-yl)phenyl)-5-(o-tolyl)-1,3,4-oxadiazole (25)

Pale yellow powder, yield: $0.083 \mathrm{~g}(83 \%)$; m.p.: $348.1^{\circ} \mathrm{C}$; IR $(\mathrm{KBr})\left(v_{\max }, \mathrm{cm}^{-1}\right): 3179,1614,1317,1287,1055$, 1000. ${ }^{1} \mathrm{H}$ NMR (500 MHz, DMSO) $\delta: 2.34,2.36$ (s, $6 \mathrm{H}, \mathrm{CH}_{3}-5$,6-benzimidazole), 2.73 (s, $3 \mathrm{H}, \mathrm{CH}_{3}-2$ '”-Ar), 7.34 (s, $1 \mathrm{H}, \mathrm{Ar}), 7.52-7.44(\mathrm{~m}, 3 \mathrm{H}, \mathrm{Ar}), 7.56(\mathrm{td}, 1 \mathrm{H}, J=7.5,1.1 \mathrm{~Hz}, \mathrm{Ar}), 8.13(\mathrm{~d}, 1 \mathrm{H}, J=7.6 \mathrm{~Hz}, \mathrm{Ar}), 8.28(\mathrm{~d}, 2 \mathrm{H}, J=$ $8.5 \mathrm{~Hz}, \mathrm{Ar}), 8.38$ (d, 2H, $J=8.4 \mathrm{~Hz}, \mathrm{Ar}), 12.88$ (s, 1H, NH). HR-ESI-MS: m/z $380.2535\left(\mathrm{M}^{+}\right)$.

4-(5-(4-(5,6-dimethyl-1H-benzo[d]imidazol-2-yl)phenyl)-1,3,4-oxadiazol-2-yl)phenol (26)

Pale yellow powder, yield: $0.079 \mathrm{~g} \mathrm{(79 \% );} \mathrm{m.p.:} 335^{\circ} \mathrm{C}$; IR $(\mathrm{KBr})\left(v_{\max }, \mathrm{cm}^{-1}\right): 3200,1609,1315,1263,1079,1016$. ${ }^{1} \mathrm{H}$ NMR (500 MHz, DMSO) $\delta: 2.34$ (s, 6H, $\mathrm{CH}_{3}-5,6$-benzimidazole), 7.00 (d, 2H, $J=8.5 \mathrm{~Hz}, \mathrm{Ar}$ ), 7.41 (s, 2H, Ar), $8.01(\mathrm{~d}, 2 \mathrm{H}, J=8.5 \mathrm{~Hz}, \mathrm{Ar}), 8.25$ (d, 2H, $J=8.3 \mathrm{~Hz}, \mathrm{Ar}), 8.36$ (d, 2H, $J=8.3 \mathrm{~Hz}, \mathrm{Ar}), 10.40$ (s, 1H, Ar). HR-ESIMS: $\mathrm{m} / \mathrm{z} 382.2328\left(\mathrm{M}^{+}\right)$.

2-(4-(5,6-dimethyl-1H-benzo[d]imidazol-2-yl)phenyl)-5-(pyridin-3-yl)-1,3,4-oxadiazole (27)

Pale yellow powder, yield: $0.073 \mathrm{~g}(73 \%)$; m.p.: $327.9^{\circ} \mathrm{C}$; IR $(\mathrm{KBr})\left(v_{\max }, \mathrm{cm}^{-1}\right): 3326,1611,1565,1317,1257$,

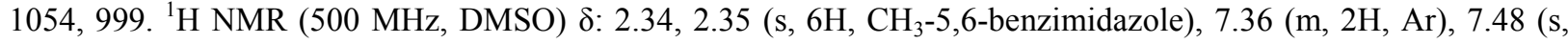
$1 \mathrm{H}, \mathrm{Ar}), 8.00$ (t, 1H, $J=4.6 \mathrm{~Hz}, \mathrm{Ar}), 8.25$ (d, 2H, $J=8.4 \mathrm{~Hz}, \mathrm{Ar}), 8.37$ (d, 2H, $J=8.4 \mathrm{~Hz}, \mathrm{Ar}), 12.91$ (s, $1 \mathrm{H}, \mathrm{NH})$. HR-ESI-MS: $\mathrm{m} / \mathrm{z} 367.1932\left(\mathrm{M}^{+}\right)$. 


\section{Norizan et al: SYNTHESIS AND CHARACTERIZATION OF OXADIAZOLE DERIVATIVES FROM BENZIMIDAZOLE}

2-(4-(5,6-dimethyl-1H-benzo[d]imidazol-2-yl)phenyl)-5-(m-tolyl)-1,3,4-oxadiazole (28)

Pale yellow powder, yield: $0.079 \mathrm{~g}(79 \%)$; m.p.: $315.4^{\circ} \mathrm{C}$; IR $(\mathrm{KBr})\left(v_{\max }, \mathrm{cm}^{-1}\right): 3198,1612,1314,1274,1080$,

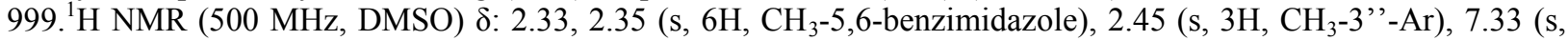
$1 \mathrm{H}, \mathrm{Ar}), 7.48$ (d, 2H, $J=9.3 \mathrm{~Hz}, \mathrm{Ar}), 7.54$ (t, $1 \mathrm{H}, J=7.6 \mathrm{~Hz}, \mathrm{Ar}), 7.97$ (d, 1H, $J=7.6 \mathrm{~Hz}, \mathrm{Ar}), 8.00$ (s, $1 \mathrm{H}, \mathrm{Ar}$ ),

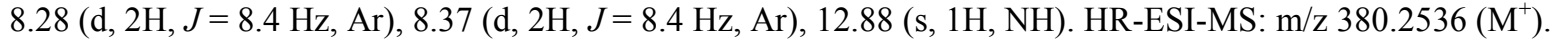

The synthesis of the target compounds began with the synthesis of sodium metasulfite adduct according to the literature protocol [5]. The resulting sulfite adduct was refluxed with 4,5-dimethyl- $O$-phenylenediamine in DMF for $6 \mathrm{~h}$ to give the arylester substituted benzimidazole. The benzohydrazide of benzimidazole was formed by refluxing arylester of benzimidazole with methanolic hydrazine hydrate (Scheme 1). The synthesis of benzimidazole benzohydrazide Schiff bases (1-14) was accomplished by reacting different aldehydes with benzimidazole benzohydrazide in $n$-butanol in the presence of a catalytic amount of acetic acid as shown in Scheme-1. A series of new 2, 5-disubstituted-1,3,4-oxadiazoles (Table 1) have been accomplished in excellent yields by the oxidation of of various aryl aldehydes with one equivalent of iodobenzenediacetate (IBD) in DMF. Compounds 15-28 were synthesized based on the reported procedure.
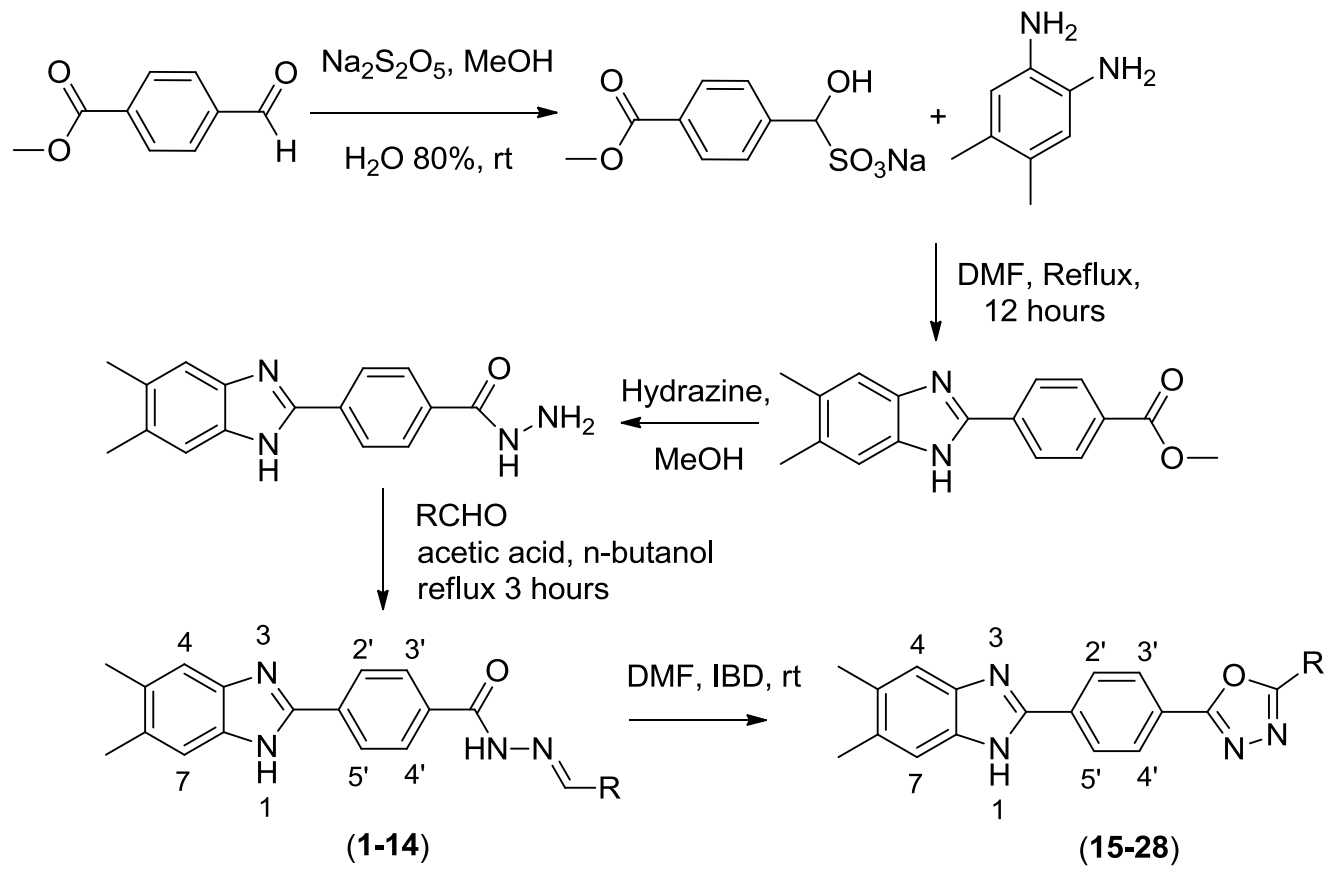

Scheme 1. Synthesis of oxadiazole derivatives from benzimidazole benzoyl hydrazide 
Table 1. Benzimidazole benzohydrazide bearing oxadiazole derivatives

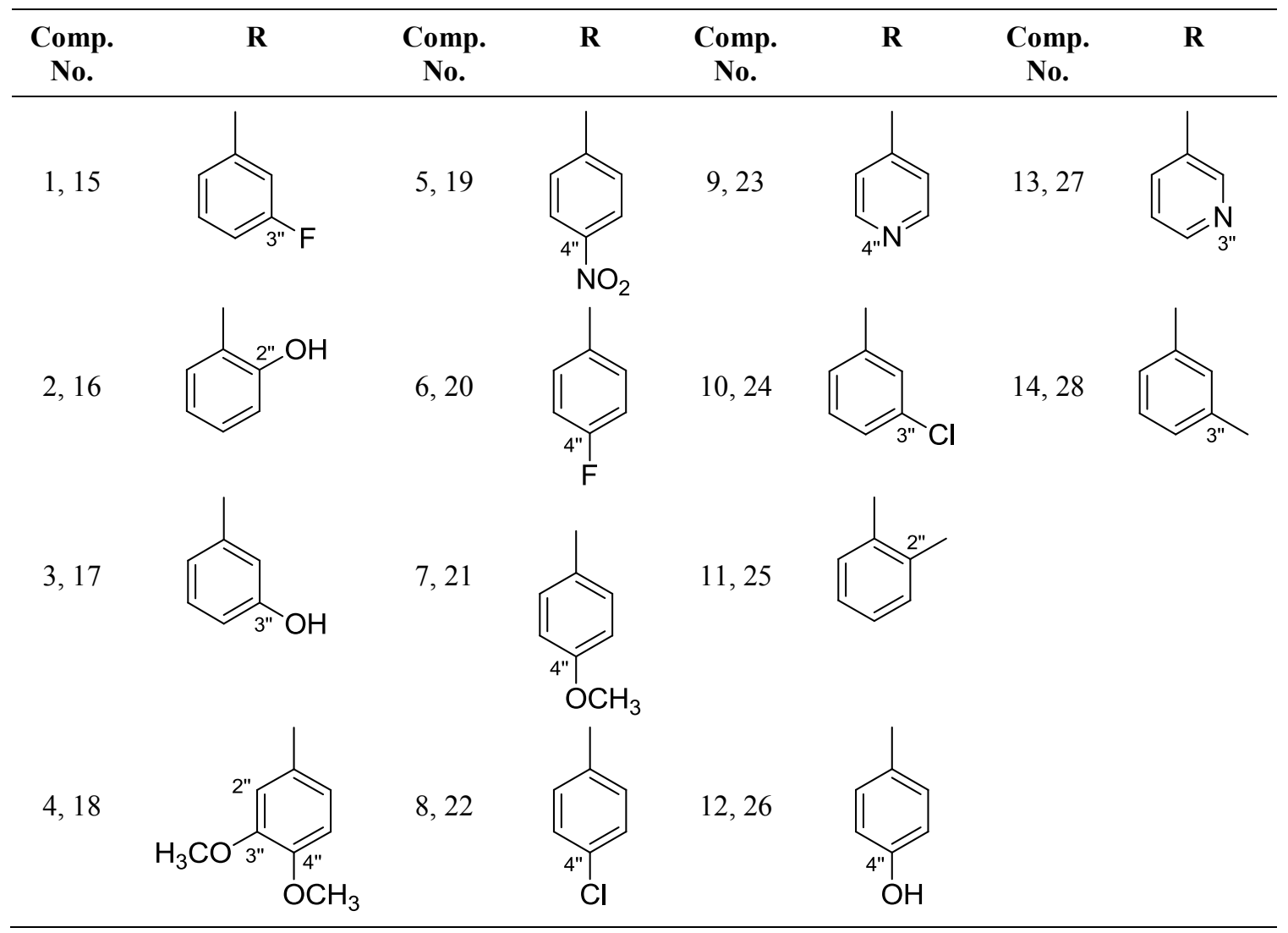

The IR spectra of the synthesized compounds exhibited characteristic absorption bands at $1600-1700 \mathrm{~cm}^{-1}$ and $3100-3300 \mathrm{~cm}^{-1}$ due to carbonyl or $\mathrm{C}=\mathrm{N}$; and $\mathrm{NH}$ group, respectively. The ${ }^{1} \mathrm{H}$ NMR spectra of compounds 1-14 showed two singlets due at $\mathrm{N}=\mathrm{CH}$ and $\mathrm{NH}$, respectively. The structure of all compounds was confirmed by their spectral data (IR, ${ }^{1} \mathrm{H}$ NMR, LCMS) and melting point. The characterization of products 15-28 was based upon a careful comparison of their IR and ${ }^{1} \mathrm{H}$ NMR spectra with those of 1-14. IR spectra of 15-28 were found to be transparent in the region of NH stretch and CO stretch. In ${ }^{1} \mathrm{H}$ NMR spectra of 15-28 the disappearance of their singlet due to $\mathrm{N}=\mathrm{CH}$ and $\mathrm{NH}$ proton confirms the oxidation of 1-14 into 15-28.

\section{Conclusion}

A series of new 2,5-disubstituted-1,3,4-oxadiazoles derivatives of benzimidazole (15-28) were synthesized in good yield and characterized by ${ }^{1} \mathrm{H}$ NMR, IR, MS spectroscopic methods and melting point. A further study to acquire information concerning the pharmacological activity is in progress.

\section{Acknowledgement}

The authors would like to acknowledge Faculty of Pharmacy, Universiti Teknologi MARA Puncak Alam, Ministry of Education Malaysia and Universiti Teknologi MARA for the financial support under RAGS grant 600RMI/RAGS/5/3/ (2/2012).

\section{References}

1. Subudhi, B. B., Panda, P. K., Kundu, T., Sahoo, S. and Pradhan, D. J. (2007). Synthesis and biological evaluation of benzimidazole and thiazolidinone derivatives. Pharmaceutical Research, 6 (2): $114-118$. 


\section{Norizan et al: SYNTHESIS AND CHARACTERIZATION OF OXADIAZOLE DERIVATIVES FROM}

BENZIMIDAZOLE

2. Afaf, H. E., Fanmy, H. H. and Abdelwad, S. H. (2000). Synthesis and antimicrobial activity of some new benzimidazole derivatives. Molecules, 5(12): $1429-1438$.

3. Ozden, S., Karatas, H., Yildiz, S. and Goker, H. (2004). Synthesis and potent antimicrobial activity of some novel 4- (5,6-Dichloro-1H-benzimidazol-2-yl)-N-substituted benzamides. Archiv der Pharmazie 337: 556 562.

4. Tebbe, M. J., Spitzer, W. A., Victor, F., Miller, S. C., Lee, C. C., Sattelberg, T. R., McKinney, E. and Tang, J. C. (1997). Antirhino/enteroviral vinylacetylene benzimidazoles: a study of their activity and oral plasma levels in mice. Journal of Medicinal Chemistry, 40 (24): 3937 - 3946.

5. Migawa, M. T., Girardet, J. L., Walker, J. A., Koszalka, G. W., Chamberlain, S. D., Drach, J. C. and Townsend, L. B. (1998). Design, synthesis, and antiviral activity of alpha-nucleosides: D- and L-isomers of lyxofuranosyland (5-deoxylyxofuranosyl) benzimidazoles. Journal of Medicinal Chemistry, 41 (8): 1242 - 1251.

6. Sidwell, R. W. and Huffman, J. H. (1971). Use of disposable micro tissue culture plates for antiviral and interferon induction studies. Applied Microbiology, 22 (5): 797 - 801.

7. Aydin, S., Beis, K. and Can, D. D. (2003). Analgesic and antispasmodic activities of 2-(2-nitro-phenyl)-1Hbenzimidazole 5-carboxylic acid: evidence for the importance of the 2-(O-substituted phenyl) group. Pharmazie, 58: 405 - 408 .

8. Kumar, V. R., Vaidya, S. D., Kumar, B. V. S., Bhise, U. N., Bhirud, S. B. and Mashelkar, U. C. (2008). Synthesis, anti-bacterial, anti-asthmatic and anti-diabetic activities of novel $N$-substituted-2-(4-phenylethynylphenyl)-1 $H$-benzimidazoles and $N$-substituted 2[4-(4,4-dimethyl-thiochroman-6-yl-ethynyl)-phenyl)-1 $H$ benzimidazoles. European Journal Medicinal Chemistry, 43 (5): 986 - 995.

9. Padmavathi, V., Reddy, G. S., Padmaja, A., Kondaiah, P. and Shazia, A. (2009). Synthesis, antimicrobial and cytotoxic activities of 1,3,4-oxadiazoles, 1,3,4-thiadiazoles and 1,2,4-triazoles. European Journal of Medicinal Chemistry, 44 (5): $2106-2112$.

10. El-Emam, A. A., Al-Deeb, O. A., Al-Omar, M. A. and Lehmann, J. (2004). Synthesis, antimicrobial, and antiHIV-1 activity of certain 5-(1-adamantyl)-2-substituted thio-1,3,4-oxadiazoles and 5-(1-adamantyl)-3substituted aminomethyl-1,3,4-oxadiazoline-2-thiones. Bioorganic and Medicinal Chemistry, 12 (19): 5107 5113.

11. Kadi, A. A., El-Brollosy, N. R., Al-Deeb, O. A., Habib, E. E., Ibrahim, T. M. and El-Emam, A. A. (2007). Synthesis, antimicrobial, and anti-inflammatory activities of novel 2-(1-adamantyl)-5-substituted-1,3,4oxadiazoles and 2-(1-adamantylamino)-5-substituted-1,3,4-thiadiazoles. European Journal of Medicinal Chemistry, 42 (2): 235 - 242.

12. Sahin, G., Palaska, E., Ekizoglu, M. and Ozalp, M. (2002). Synthesis and antimicrobial activity of some 1,3,4oxadiazole derivatives. Farmaco II, 57 (7): 539 - 542.

13. Amir, M., Javed, S. A. and Kumar, H. (2007). Synthesis of some 1,3,4-oxadizole derivatives as possible antiinflammatory agents. Indian Journal of Chemistry Section B, 46: 1014 - 1019.

14. Wagle, S., Adhikari, A. V., Kumari, N. S. (2008). Synthesis of some new 2-(3-methyl-7-substituted-2oxoquinoxalinyl)-5-(aryl)-1,3,4-oxadiazoles as potential non-steroidal anti-inflammatory and analgesic agents. Indian Journal of Chemistry, 47: 439 - 448.

15. Najer, H., Giudicelli, R., Moral, C. and Menin, M. (1996). Synthesis of some 5- aryl- 2- dimethylamino-1,3,4oxadiazoles with anti -inflammatory action. Bulletin de la Societe chimique de France, 1: 153 - 159.

16. Almasirad, A., Tabatabai, S. A., Faizi, M., Kebriaceezadeh, A., Mehrabi, N., Dalvandi, A and Shafiee, A. (2004). Synthesis and anticonvulsant activity of new 2-substituted-5- [2-(2-fluorophenoxy)phenyl]-1,3,4oxadiazoles and 1,2,4-triazoles. Bioorganic and Medicinal Chemistry Letters, 14 (20): 6057 -6059.

17. Zarghi, A., Tabatabai, S. A., Faizi, M., Ahadian, A., Navabi, P. and Zanganeh, V. A. (2005). Synthesis and anticonvulsant activity of new 2-substituted-5-(2-benzyloxyphenyl)-1,3,4-oxadiazoles. Bioorganic and Medicinal Chemistry Letters, 15 (7): 1863 - 1865.

18. Burbuliene, M. M., Jakubkiene, V., Mekuskeine, G., Vdrenaite, E., Smicius, R. and Vainilavicius, P. (2004). Synthesis and anti-inflammatory activity of derivatives of 5-[(2-disubstitutedamino-6-methyl-pyrimidin-4-yl)sulfanylmethyl]-3H-1,3,4-oxadiazole-2-thiones. Farmaco II, 59 (10): 767 - 774.

19. Sengupta, P., Dash, D. K., Yeligar, C. V., Murugesh, K., Rajalingam, D., Singh, J. and Maity, T. K. (2008). Evaluation of anticancer activity of some 1,3,4-oxadiazole derivatives. Indian Journal of Chemistry Section B, 147: $460-462$. 
20. Taha, M., Ismail, N. H., Jamil, W., Rashwan, H., Kashif, S. M., Sain, A. A., Adenan, M. I., Anouar, E. H., Ali, M., Rahim, F. and Khan, K. M. (2014). Synthesis of novel derivatives of 4-methylbenzimidazole and evaluation of their biological activities. European Journal of Medicinal Chemistry, 84: $731-738$.

21. Prasanna Kumar, B. N., Mohana, K. N., Mallesha, L. (2013). Synthesis of N-[\{5-Aryl-1,3,4-oxadiazole-2yl \} methyl]-4-methoxyaniline derivatives and their anticonvulsant activity. Journal of Chemistry, $2013: 1-8$. 